weeks. The serum level of elastase remained unchanged. The administration of lactoferrin in the chronic phase of adjuvant arthritis resulted in a steady suppression of the inflammatory process (decreased hind paws swelling and local temperature, white blood cell count and erythrocyte sedimentation rate, an increase in weight gaining and a decrease in serum elastase level), which lasted until the end of experiment (day 50). This effect was achieved with lower doses of the drug $(9 \mathrm{mg} / \mathrm{kg})$, though its strength was dose-dependent. The maximal suppression of adjuvant arthritis was obtained with the repeated injections of lactoferrin (5 injections every other day with total dose of $60 \mathrm{mg} / \mathrm{kg}$ ) in the chronic phase of adjuvant arthritis (days 2331). In these rats the serum concentration of elastase was reduced down to normal level and the rate of generalisation was less than $8 \%$.

Conclusion Lactoferrin is a potent anti-inflammatory agent effective in treatment of adjuvant arthritis in rats. The maximal suppression of the inflammatory process is obtained in the chronic phase of adjuvant arthritis.

\section{AB0023 IN VITRO PERIPHERAL BLOOD MONONUCLEAR CELLS AUTOREACTIVITY AGAINST AUTOLOGOUS EPIDERMAL CELLS AND THE PRESENCE OF ANTI-SKIN AUTOANTIBODIES IN PATIENTS WITH RHEUMATOID ARTHRITIS}

IJ Hromadnikova, P Vavrincova, K Stechova, M Cermakova-Frantlova, D Chudoba, J Vavrinec. ${ }^{2}$ Nd Paediatric Clinic, University Hospital Motol, Prague 5, Czech Republic

\subsection{6/annrheumdis-2001.928}

Background We discuss the ability of peripheral blood mononuclear cells (PBMN) of patients with rheumatoid arthritis (RA) or juvenile idiopathic arthritis to damage autologous skin or lyse autologous keratinocytes if co-cultured in vitro.

We discuss a well as the presence of anti-skin anti-intercellular (ASA-IC), anti-skin anti-basement membrane (ASA-BM) and antikeratin (AKA) antibodies of IgG class in patients' sera without clinical features of vesicular bullous diseases.

\section{Objectives}

Methods A modified in vitro skin explant assay originally developed to predict acute graft versus host disease (GvHD) in allogeneic stem cell transplants was used in order to see if GvHR-like histopathological changes occur when patients' PMNC would be co-cultured with autologous skin. 51Cr release cytotoxic assay was used to prove if observed skin damage was really of an autoimmune origin. An indirect immunofluorescence test on rat, monkey and guinea pig oesophagus substrates was used for the detection and quantification of ASA-IC, ASA-BM and AKA antibodies.

Results We found out that PBMN of 29 out of 34 patients with RA or JIA $(85,3 \%)$ were able to induce histopathological changes of grade II or above in autologous skin explants. Similarly, we observed that PBMN lysed autologous keratinocytes, but not autologous lymphoblasts when co-cultured in vitro.

No skin damage or keratinocytes lysis was observed in healthy controls. Overall ASA-IC were detected in 50 out of 57 studied patients' sera samples $(87,7 \%, p=0,0003)$. No ASA-BM positivity was observed in a studied cohort. AKA were detectable in 33 out of 60 patients $(55 \%, \mathrm{p}=0,0001)$.

Conclusion We suggest that PBMN of patients with RA or JIA might recognise similar autoantigen (s) expressed on epidermal cells that give rise an autoimmune response in synovium. However we observed a high incidence of ASA-IC antibodies in patients with RA and JIA we suppose that subclinical pemphigus occurring in this group might be exacerbated with different stimulus including pemphigus inducing drugs.

\section{REFERENCES}

1 Osteolog Bull. 2000;2:70-11

2 Czech Rheumatol. 1999;4:166-74

\section{AB0024 ACTIVITIES OF PURINE METABOLISM ENZYMES AND ANTIOXIDANT SYSTEM IN RHEUMATOID ARTHRITIS PATIENTS}

AB ZZborovsky, VF Martemyanov, MY Stazharov, SA Bedina, TP Chernykh, EE Mozgovaya. Laboratory for Clinical Biochemistry, Research Institute for Clinical and Experimental Rheumatology, Volgograd, Russia

\subsection{6/annrheumdis-2001.929}

Background The results of Antioxidant system researches of blood at Rheumatoid arthritis are inconsistent and not enough attention was given to Purine metabolism questions.

Objectives The improvement of Rheumatoid arthritis diagnostics with use of the enzymatic tests.

Methods 71 RA patients and 30 healthy controls were under observation. Adenosin deaminase (ADA), AMP-desaminase (AMPDA), Guanin desaminase (Guanase), Xanthine oxidase (XO), Xanthine dehydrogenase (XDG) activities were determined in blood serum; Glutation peroxidase (GP), Glutation reductase (GR), Superoxid dismutase (SOD) activities ? in plasma (pl.) and erythrocytes (er.). ADA, XDG, SOD isoenzymes were determined also. The enzymatic indices were defined in the process of treatment thrice and were analysed depending on clinical specificity of the disease.

Results Increased ADA, Guanase, GRpl., GPer., XO, XDG, SOD activities, decreased AMPDA, GRer., GPpl. activities, increase ADA-2, XDG-2, SOD-1 isoenzymes were observed in RA patients (I degree of activity) in comparison with healthy controls. The increase of the pathological process activity was accompanied with the increase of Guanase, GRpl., XO, SODpl. activities, ADA-2, XDG-2, SOD-1 isoenzymes and the decrease of ADA, XDG, GRer. activities. RA patients with system defeats have more increased AMPDA, Guanase, XO activities, ADA-2, XDG-2 isoenzymes, but less ADA, XDG activities in comparison with patients, which had arthritis form of RA. The system defeates were characterised with the decrease of SOD, GPer. activities and the increase of GPpl. activity; patients with the arthritis form of RA had contrary changes. We observed more increase AMPDA, Guanase, XO, ADA-2 activities and less ADA activity in fast progressing RA in comparison with slow progressing.

Conclusion The enzymatic indices essentially depended on clinical specificity of the disease and can be used as additional diagnostic criterions in RA. 